\title{
ANALYSIS OF THE LATEST
}

\author{
AUSTROADS GUIDELINES
}

FOR

FITNESS TO DRIVE

\section{AS PROMULGATED IN MARCH 2012}

\author{
ROY G BERAN \\ Professor, School of Medicine, Griffith University \\ Conjoint Associate Professor, University of New South Wales \\ Consultant Neurologist and Director, Strategic Health Evaluators \\ Mailing Address: Suite 5, Level 6 \\ 12 Thomas St \\ Chatswood NSW 2067 \\ Phone: 0294114991 \\ Fax: 0294131353 \\ Email: roy.beran@unsw.edu.au
}

Word Count (Main Text): 2,751 Words

Word Count (Abstract): 127 Words 


\section{INTRODUCTION}

Patients consider driving a right, not a privilege. Preventing driving is traumatic ${ }^{1}$ provoking potential conflict. Some patients perceive doctors as adversaries rather than advocates $^{2}$. Previous guidelines were unclear, created ambiguity and possible confusion $^{3}$. Consultants may overtly support guidelines while acting contrary to them ${ }^{4}$. Case law has generated concern" ${ }^{5}$. Without mandatory reporting, “...physicians can still be found liable if they fail to report a patient who is later determined to have caused harm to others as a result of a medical impairment behind the wheel..."6.

Younger drivers display adverse behaviour with speeding and intoxication ${ }^{7}$ while older drivers have physical, visual and cognitive impairments ${ }^{7}$. Driving demands timely and appropriate reaction to unforeseen circumstances ${ }^{8}$. Guidelines must be sensitive and responsive to latest developments. Austroads guidelines ${ }^{9}$ were first published in 1998 , with further editions in 2001, 2003 (reprinted in 2006) and $2012^{9}$.

The disclaimer remains, “...Neither Austroads nor the authors accept responsibility for any consequences arising from their (guidelines) application ... Health professionals should maintain an awareness of any changes in health care ... that may affect their assessment ... (and) ... maintain an awareness of changes in the law ...".

The latest guidelines, while endorsed by seven professional bodies, named additional organisations, which contributed but did not endorse them, raising questions concerning legitimacy. The guidelines state: “...Where there are concerns ... advice may be sought from the professional's Medical Defence Organisation or legal advisor...", suggesting potential vulnerability. 


\section{BACKGROUND}

Data from the preceding year report “...1,367 Australians were killed on our roads and many tens of thousands seriously injured. The total economic cost of this exceeds $\$ 15$ billion annually and the accompanying social costs greatly impact on our communities ...", necessitating robust health standards.

Some see doctors as the 'gate-keepers'2 provoking 'doctor-shopping'2 or concealing information causing reduced safety ${ }^{10}$. Regulations should consider scientific evidence not 'gut feelings' 11,12 . Research confirms comparable US fatalities between states requiring $6-12$ months seizure-freedom or only 3 months ${ }^{13}$, with mounting physician pressure to liberalise restrictions for conditions, such as epilepsy or neuromuscular disease ${ }^{14,15}$. The new guidelines tackle some of these concerns with enhanced scientific basis.

\section{PART A}

The guidelines aim to: increase road safety with patient assessment responsive to medical data; standardise conditional licensing; promulgate understanding of obligations; and disseminate information. They review the impact of medical conditions on driving and mandate updated medical knowledge and appreciation of legislation and responsibilities. They expect drivers to: self report, be truthful, compliant and adhere to conditional licences (including periodic reviews).

Health professionals should counsel patients on: fitness to drive; impact of medical conditions; responsibility to self-report; manage conditions affecting driving; and determine patients' suitability for conditional licences. 
Licensing authorities must determine all licensing decisions; evaluate appropriate reports; arbitrate conditional licence applications; consider recommendations and other factors; and provide driver education and information.

Confidentiality, recognised as integral to best practice, is restricted to enhance safety. The guidelines expect reporting of patients who: cannot appreciate the impact of their condition; cannot appreciate advice; drive contrary to advice; and endanger public safety. South Australia and Northern Territory mandate reporting while the remainder indemnify reporting in good faith without consent.

Caution is advised for new patients because of 'doctor shopping' with specialist opinion encouraged for dubious cases, especially commercial drivers, allowing leniency if specialist access is limited.

Private standards apply to small vehicles, unless used for commercial purposes, including fee-paying passengers or dangerous goods. Commercial standards encompass larger vehicles and those for paying passengers, long-haul drivers and specific industries, including driving instructors. Commercial drivers require: longer non-driving qualifying period; specialist evaluation; exclusion of conditions like dementia; and imposition of shorter review periods. Conditional licences allow driving with additional requirements to optimise safety and quality of life. If required, specialist opinion must be pertinent to that specialist's expertise.

The guidelines describe circumstances affecting driving, including: driver experience; physical and mental capacity; traffic and road conditions; legal rules; environmental factors, including light and weather; vehicle determinants and maintenance; personal factors, with time pressures; and potential for distraction. Commercial drivers also 
have: rosters and contractual imperatives; additional expectations; legal obligations; vehicle considerations, including load distribution; passenger requirements and dangers; transporting dangerous goods; and driver fatigue with shift work and prolonged driving.

Specific conditions influencing driver safety include: blackouts; cardiovascular disease; diabetes; musculoskeletal conditions; neurological conditions; psychiatric conditions; substance misuse and dependency; sleep disorders; and vision problems. Some conditions are self-limiting, such as post-major-surgery, thereby avoiding notification but still imposing temporary driving restriction. Multiple co-morbidities necessitate value judgments per condition and overall assessment of combined effects, with appraisal of: general functionality; additional reported information; disease progression; clinical findings and impact on driving.

Comparing September 2003 and March 2012 guidelines, the purpose of the guidelines has been expanded beyond "medical practitioners and drivers" to include "health professionals, driving licensing authorities and drivers", satisfying the wider definitions of the Australian Health Practitioner Regulation Agency. It also acknowledges that licensing authorities must also meet responsibilities. The new guidelines removed reference to providing "... a primary source of criteria for State and Territory Driving Licensing Authority in assessing fitness to drive ..." (at 2, 2003; at 3, 2012) thereby suggesting possible need for wider scientific referencing for decision-making.

The background information, within the new guidelines, has been expanded with greater discussion regarding their development and emphasising evidence-based consideration (at 5, 2012). Greater emphasis has acknowledged potential patient 
animosity at possible driving restrictions and the need to circumvent 'doctor shopping' (at 9, 2012).

The role of the general practitioner has been subtly reinforced by newly adopted language, such as "... In the case of commercial vehicle drivers, the opinion of a medical specialist is required for recommendation ..." (at 23, 2003). The modified language includes, "... is generally required for initial recommendation ..." (at 9, 2012). The new guidelines also make allowance for delayed specialist consultation, especially for peripheral neuropathy, rheumatoid arthritis and type II diabetes (at 15, 2012). They are less accommodating for conditions such as ischaemic heart disease, sleep apnoea or "blackouts", although a degree of ambiguity does arise. There also appears a highlighted "blue box" reference in commercial drivers, advocating the use of "video conferencing" (at 15, 2012).

A new entry concerning reinstating licences or removing conditions (at 15, 2012) recognises the possible need for "... the driver/applicant to undertake and pass an onroad evaluation ...".

The section on "Multiple Conditions and Age-Related Change" represents significant expansion (at 22, 2003; and 19 - 20, 2012) including consideration of "capacity to learn to drive", "occupational therapy assessment" and "on-road driving assessment". A further "blue box" highlights that health professional's need to fully evaluate risk management.

Another timely addition is the discussion of "Drugs and Driving" (at $20-21,2012$ ) with specific reference to benzodiazepines, antidepressants, antipsychotics and opioids. "Information and Assistance for Patients" is a further addition (at 23, 2012) both 
advising health professionals to be empathic and referring patients to self-help organisation while providing useful references.

\section{PART B}

Three examples (blackouts, epilepsy and sleep disorders) demonstrate the complexities of Part B.

Syncope and blackouts were grouped together and treated only superficially in the 2003 guidelines (at 92 - 93) with syncope dictating two months driving restrictions for private drivers and six months for commercial drivers. Ambiguity arose when "syncope" was also addressed under the rubric of "Cardiovascular Disease", requiring only three months driving restrictions for both categories (at 37, 2003).

This was an inadequate, ambiguous and illogical direction lacking scientific integrity and has been vastly improved in the 2012 guidelines (at $33-34,2012$ ). There is now up to 10 years driving restriction for multiple blackouts for commercial drivers. The pathophysiological basis of "blackouts" underpins any imposed restriction on driving, acknowledging the lack of clarity with such terminology as "blackouts".

'Blackouts' cause “... transient impairment or loss of consciousness...”, including: vasovagal syncope (faint); cardiovascular syncope; and other causes, such as metabolic disorders, intoxication or sleep disorders. 'Blackouts' preclude driving until aetiology is determined. Some may claim 'blackout' to excuse offences, such as inappropriate phone use. Where aetiology defies diagnosis, epilepsy-related restrictions apply. Vasovagal syncope allows continued driving; cardiovascular causes evoke cardiac restrictions; epilepsy invokes epilepsy restrictions; hypoglycaemia 
demands diabetic restrictions; drug and/or alcohol abuse imprecates substance abuse rules; sleep disorders impose sleep considerations; and uncertainty defaults to epilepsy.

Private drivers, experiencing 'blackout', may hold conditional licences with: single blackout or all episodes within 24-hours, no episodes for 6-months and annual review; $>1$ episode, separated by $>24$-hours require 12-months restriction with specialist assessment. Commercial drivers need 5-years episode-freedom for single 'blackout' or all within 24-hours and 10-years for $>1$ 'blackout' over $>1$-day. Specialists may suggest shorter review periods.

The 2003 guidelines considered epilepsy a separate entity (at 55 - 60) with other neurological conditions, excluding epilepsy and syncope, assessed separately and included dementia and cognitive impairment, despite the next section of the 2003 guidelines identified as Older Drivers (at 76 - 78). The 2003 material, considering the elderly in isolation, was so superficial and unhelpful that it served no useful purpose other than to remind assessors of problems affecting advanced age. It did not mention Alzheimer's Disease.

Part B6, "Neurological Conditions" was significantly enhanced (at 70 - 99, 2012). Dementia attracted six lines of discussion in 2003 (71) and four pages in 2012 (71 74). Conversely the elderly (at $76-78,2003$ ) is no longer considered separately in the 2012 guidelines but is encompassed under those conditions which affect the elderly, rather than being age discriminatory.

In the epilepsy specific subsection of neurological disorders (section B6.2 "Seizures and Epilepsy") the space devoted to background discussion has doubled (at 55 - 56, 
2003; at 75 - 78, 2012) with altered emphasis. "Default standard", as the common restriction against which modification may apply in certain circumstances, has been introduced. Each of the exceptions to the default standard is discussed in detail (77 $78,2012)$ providing much greater information for those less conversant with epilepsy than was the case in 2003 (at 56).

Seizures may range from subjective sensations to convulsions. Non-compliance doubles accident rates. Well-managed, seizure-free private drivers may hold conditional licence if they: appropriately manage their epilepsy; are compliant; maintain medical contact; and undergo regular reviews.

Default standards apply to all seizures, except: childhood-only seizures; first seizures; epilepsy treated for the first time; acute symptomatic seizures; 'safe' seizures; sleeponly seizures; seizures in previously well-controlled epilepsy; and exceptional situations, with specialist advice. Patients with childhood febrile seizures and benign childhood epilepsy, seizure-free after age 11-years, can drive without restriction.

About half first seizures do not recur. Epilepsy first treated within the preceding 18months, seizure-free after achieving standard regime or 6-months following treatment initiation, can drive after 6-months. A further seizure, 3-months after treatment commences, within 6-months tolerance, can drive within 9-months of treatment commencing. Second seizure, 7-months following treatment initiation, 1-month beyond the 6-months, reverts to default period. Seizures, caused by transient brain disorder, cannot drive until the relative risk becomes acceptable, commercial drivers requiring specialist advice. Seizure recurrence, after initial causative illness resolves, even with second bout thereof, removes transient disorders as applicable. A seizure with encephalitis, and recurrence following recovery, before commencing medication, 
evokes 'epilepsy treated for the first time'. Seizures post two separate benzodiazepine withdrawals invoke default criteria.

Seizures, not affecting consciousness or capacity, demand corroboration from reliable witness(es) or video EEG because patients may not appreciate impaired performance. Private drivers with time-specific seizures, upon waking, may have restricted licences, indicating permissible times. 'Auras' may permit driving once established without exception, reliant upon witness(es) or video EEG and if exceptionally well-documented.

Sleep-only seizures, without awake-seizures, permit private driving. Previous 'awake' seizures increase the restriction period. Private drivers with a solitary seizure $(>12-$ months seizure-freedom) may have reduced seizure-free period, depending on seizure provocation cause. Sleep deprivation, missed medication, over-the-counter medications, alcohol or acute illness provocation permits private driving upon review. Other circumstances include: epilepsy treated by surgery; medication non-compliance; medication cessation; seizures causing a crash; or resumption of an unconditional licence. Epilepsy surgery may stop seizures, allowing driving after prescribed seizurefree period unless causing visual field defects. Treatment cessation imposes restrictions and suspected non-compliance necessitates blood level monitoring. Previous compliance, with $\geq 1$-year seizure freedom, and solitary seizure after missing medication dosage, is handled as '....seizure in a person whose epilepsy was previously well controlled...'.

Medication withdrawal, following medical advice, precludes driving until 3-months after cessation. Adverse event provoked reduction avoids restrictions with only adverse event reduction applicable to commercial drivers. 'Seizure-related crash' increases risk evoking the default period. 
Seizure-freedom for $\geq 5$-years off medications for $\geq 12$-months permits an unconditional private (not commercial) licence. Default periods apply to other seizures excluding above-cited exceptions.

Commercial drivers may hold conditional licences with at least annual review, pending epilepsy specialist opinion if: $\geq 5$-years seizure-free (with or without medication); EEG shows no epilepsy; and default commercial standards apply. No reduction in seizurefree period for commercial drivers applies unless in exceptional circumstances with specialist endorsement. Shorter restrictions apply to private drivers with: $\geq 2$-years 'safe' seizures; $\geq 12$-months sleep-only seizures (no awake-seizures); $\geq 2$-years sleeponly seizures ( $\geq 2$-years no awake-seizures); previously well-controlled epilepsy with seizure caused by identified, avoidable and previously unencountered provocateur with $\geq$ 4-weeks seizure-freedom; previously well-controlled epilepsy without identified cause for solitary additional seizure and $\geq 3$-months seizure-freedom; $\geq 12$-months seizurefreedom post-epilepsy surgery; or in exceptional cases upon medical advice.

While neurological consideration expanded (incorporating: dementia; seizures and epilepsy; vestibular disorders; and other neurological and neuro-developmental conditions) (at 70 -99, 2012), allocation of space for sleep disorders has not increased (87 - 91, 2003; 105 - 109, 2012) and the latest guidelines have provided fewer references (38 in 2003; 12 in 2012) with no suggested 'further readings' offered in 2012 (2 cited in 2003, at 91). The Epworth Sleepiness Scale is delineated in 2012 (at 106) with introduction to other tools, not discussed in 2003, such as the maintenance of wakefulness test and the multiple sleep latency test (at 106, 2012). Referral to a specialist sleep physician is strongly recommended in the latest guidelines. 
Sleep disorders cause 2 - 7 times the general population crash rate, comparable with alcohol impairment or sleep deprivation. Sleep apnoea $(R D I \geq 34)$ increases crash rates. Shift work and sleep deprivation exacerbate crash risks.

CPAP for sleep apnoea reduces daytime sleepiness and crash risk compatible with controls. Mandibular advancement devices improve daytime sleepiness but no studies confirm crash reductions.

Increased daytime sleepiness may result from: sleep deprivation; poor sleep hygiene, irregular sleep patterns; sedatives; or alcohol. Insufficient sleep $(<5$-hours) before driving increases crash rates. Excessive daytime sleepiness relates to: sleep apnoea; nocturnal hypoventilation; periodic limb movement (especially with arousal); circadian rhythm disorders; insomnia; and narcolepsy.

Higher risk occurs with: excessive daytime sleepiness (Epworth Sleepiness Scale 16 24); frequent self-reported driver sleepiness; and previous crash(es) due to inattention or sleepiness.

Conditional licences are allowed, for private and commercial drivers, with sleep apnoea if compliant and responsive to treatment. Narcolepsy allows conditional licences for compliant private drivers under specialist supervision. Commercial drivers with narcolepsy, wanting conditional licence, must: have no cataplexy; be compliant; be symptom-free ( $\geq 6$-months); have normal latency with Mean Wakefulness Test.

\section{DISCUSSION}


Previous guidelines contained language problems, ambiguity and potential confusion ${ }^{3}$. Much has been rectified, with greater respect for science, enhanced clarity and reduced ambiguity. Driver evaluation is pivotal ${ }^{16}$ and these guidelines are more 'userfriendly'. Not all contributing to the guidelines endorsed them but they do offer significant improvement.

The disclaimer still obviates author responsibility, consequent to application. Reference to Medical Defence Organisations implies the guidelines impose vulnerability irrespective of indemnity with demand for currency regarding health care and legislation.

While defining driver and licensing authorities' responsibilities, health professionals remain pivotal for evaluation, with professionals expected to report at-risk drivers and vigorously apply the guidelines. A subtle but significant change in language in Part A has increased the potential burden for General Practitioners.

Acknowledging the doctor-patient relationship and confidentiality, the guidelines dictate notification of recalcitrant patients, irrespective of consent. While mandatory in Northern Territory and South Australia, despite reluctance to report ${ }^{4}$, professionals must report where applicable and appreciate 'doctor-shopping', especially for commercial drivers.

Part A broadly reviews the nature, relevance and importance of the guidelines while Part B examines identified conditions of which three have been discussed in detail to demonstrate how the guidelines have evolved and to emphasise the impact of those changes. Health professionals should read the guidelines carefully as road safety is 
paramount. This is especially relevant to specialist physicians who may need to provide additional input regarding their particular area of expertise. 


\section{REFERENCES:}

1. Molnar FJ, Byszewski AM, Marshall SC, Man-Son-Hing M. In-office evaluation of medical fitness to drive: practical approaches for assessing older people. Can Fam Physician 2005; 51: 372 - 379.

2. Somerville ER, Black AB, Dunne JW. Driving to distraction - certification of fitness to drive with epilepsy. Med J Aust 2010; 192 (6): 342 - 344.

3. Beran RG. Analysis and overview of the guidelines for assessing fitness to drive for commercial and private vehicle drivers. Intern Med J 2005; 35 (6): 364 368.

4. Beran RG, Ainley LA, Beran ME. Opinions, attitudes and practices of Australian neurologists with regard to epilepsy and driving. Intern Med J 2007; 37 (4): 251 -257 .

5. Beran RG, Gerber P, Devereux JA. Usefulness of Austroads' fitness-to-drive guidelines: lessons from the Gillett case. Med J Aust 2009; 190 (9): 503 - 505.

6. Molnar FJ, Simpson CS. Approach to assessing fitness to drive in patients with cardiac and cognitive conditions. Can Fam Physician 2010; 56 (11): 1123 1129.

7. Marshall SC.The role of reduced fitness to drive due to medical impairments in explaining crashes involving older drivers. Traffic Inj Prev 2008; 9 (4): 291 298. 


\section{(References continued):}

8. Austey KJ, Wood J, Lord S, Walker JG. Cognitive, sensory and physical factors enabling driving safety in older adults. Clin Psychol Rev 2005; 25 (1): 45 - 65.

9. Austroads \& National Transport Commission (NTC) Australia Assessing fitness to drive for commercial and private vehicle drivers: medical standards for licensing and clinical management guidelines [Internet]. Sydney: Austroads; 2012 March [cited 2012 Apr 4]. 176p. Available from:

https://www.onlinepublications.austroads.com.au/items/AP-G56-12

10. Salinsky MC, Wegener K, Sennema F. Epilepsy, driving laws, and patient disclosure to physicians. Epilepsia 1992; 33 (3): 469 - 472.

11. Drazkowski JF, Neiman ES, Sirven JI et al. Frequency of physician counselling and attitudes towards driving motor vehicles in people with epilepsy: comparing a mandatory-reporting with a voluntary-reporting state. Epilepsy Behav 2010; 19 (1): $52-54$.

12. Krumholz A. Driving issues in epilepsy: past, present, and future. Epilepsy Curr 2009; 9 (2): $31-35$.

13. Sheth SG, Krauss G, Krumholz A, Li G. Mortality in epilepsy: driving fatalities vs other causes of death in patients with epilepsy. Neurology 2004; 63 (6): 10021007. 
(References continued):

14. Bilban M. Epilepsy and driving capability in Slovenia. Seizure 2008; 17 (5): 465 $-468$.

15. Gomez- Talegon MT, Fierro I, Vicondoa A et al. Fitness-to-drive assessment in drivers with neurological and neuromuscular disorders at the driving test centres. Rev Neurol 2007; 45 (9): 526 - 531.

16. Odell M. Assessing fitness to drive: part 2. Aust Fam Physician 2005; 34 (6): $475-477$. 